\title{
TRIANGULATIONS IN MÖBIUS GEOMETRY
}

\author{
FENG LUO
}

\begin{abstract}
ABSTRACr. We prove that a conformally flat closed manifold of dimension at least three adimts a hyperbolic, spherical or similarity structure in the conformally flat class if and only if the manifold has a smooth triangulation so that all codimension one simplexes are in some codimension one spheres.
\end{abstract}

\section{INTRODUCTION}

It is known that in dimension three or higher a conformally flat manifold does not necessarily have constant curvature metric in the conformal class. For instance, the product of a closed hyperbolic manifold with $S^{1}$ (or the connected sum of two closed hyperbolic manifolds) has a conformally flat structure but it has no spherical, or hyperbolic, or similarity structures. The purpose of this note is to characterize those conformally flat closed $n$-manifolds $(n \geq 3)$ which admit spherical, or hyperbolic, or similarity structures in the preferred conformally flat classes.

In dimension three or higher, by Liouville's theorem, any local conformal diffeomorphism of $S^{n}$ is induced by a global Möbius transformation. Therefore, conformally flat manifolds are the same as manifolds with geometric structures modeled on the inversive geometry $\left(S^{n}, \operatorname{Mob}\left(S^{n}\right)\right) \quad(n \geq 3)$. The result of this note is based on the following observation of the inversive geometry.

Lemma 1. Given any $n+1(n-1)$-spheres $S_{1}, S_{2}, \ldots, S_{n+1}$ in the $n$-sphere $S^{n}$, one of the following assertions holds:

(a) (Euclidean) there is a point $x \in S^{n}$, such that $x \in S_{i}$ for all $i$.

(b) (Spherical) there is a fixed point free involution $\theta \in \operatorname{Mob}\left(S^{n}\right)$ such that $\theta\left(S_{i}\right)=S_{i}$ for all $i$.

(c) (Hyperbolic) there is an $(n-1)$-sphere $S^{n-1}$ such that $S^{n-1} \perp S_{i}$ for all $i$.

Since inversive geometry is the infinity of the hyperbolic geometry, the lemma can also be stated as:

Lemma 2. For any $n$ totally geodesic complete hypersurfaces $D_{1}, \ldots, D_{n}$ in $H^{n}$, one of the following holds:

(a) all $D_{i}$ 's intersect at a point in the sphere at the infinity;

(b) all $D_{i}$ 's intersect at a point in $H^{n}$;

(c) all $D_{i}$ 's are orthogonal to a totally geodesic complete hypersurface in $H^{n}$.

Received by the editors January 21, 1991.

1980 Mathematics Subject Classification (1985 Revision). Primary 51B10; Secondary 57Q15. 


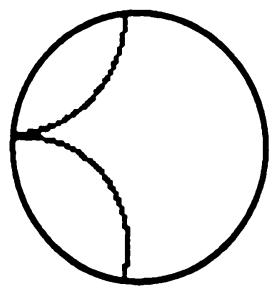

(a)

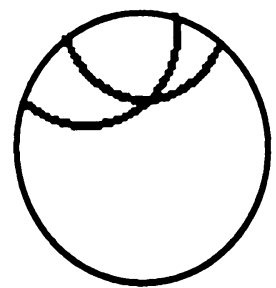

(b)

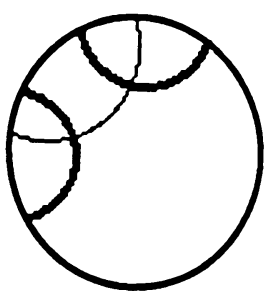

(c)

Figure 1

The major notion introduced in this note is the $n$-dimensional conformal simplex (or Möbius simplex) which is an $n$-dimensional smooth simplex in $R^{n}$ (or $\left.S^{n}\right)$ bounded by $(n-1)$-affine planes and $(n-1)$-spheres (or spheres). Applying Lemma 1, we show that conformal $n$-simplices are essentially classical geometric simplices: spherical, or Euclidean, or hyperbolic simplices. Thus, we have

Theorem. If $M^{n}$ is a closed conformally flat manifold $(n \geq 3)$ with a smooth triangulation $K$ such that all $n$-simplices in $K$ lie in some conformally flat coordinate charts and are conformal $n$-simplices, then $M^{n}$ admits a unique spherical, or similarity, or hyperbolic structure in the preferred conformally flat class.

The converse of this theorem is well known, namely, any hyperbolic or spherical (or similarity) manifold has a triangulation in which all simplices are totally geodesic (or rectilinear with respect to the similarity structure).

It seems the interesting case remains is the Möbius polyhedrons and cell decompositions of Möbius manifolds.

The organization of this note is as follows. In $\S 1$, we establish some elementary properties of the inversive geometry and conformal simplices. The theorem is proven in $\S 2$. In the last section, two proofs of Lemma 1 are given. The second proof is due to Robert Edwards.

\section{Preliminaries}

The basic model for the inversive geometry (or the conformally flat geometry in dimension greater than 2) is $\left(S^{n}, \operatorname{Mob}\left(S^{n}\right)\right)$, where $S^{n}$ is the unit sphere $\left\{x \in E^{n+1} \mid\|x\|=1\right\}$ in the Euclidean space $E^{n+1}$, and $\operatorname{Mob}\left(S^{n}\right)$ is the group of all angle preserving self-diffeomorphisms of $S^{n}$. The $i$-spheres (or geometric $i$-spheres) in $S^{n}$ are the transverse intersections of $S^{n}$ with $(i+1)$-dimensional affine space in $E^{n+1}$. For instance, a 0 -sphere is a two-point set. Another useful model for the inversive geometry is obtained by stereographic projecting $S^{n}$ with respect to any given point $x \in S^{n}$ to the Euclidean space $E^{n}$ (sending $x$ to the infinity). We will use $E^{n}$ to denote the model and simply refer it to be the Euclidean model of $S^{n}$ with $x$ as the infinity. Under the stereographic projection, an $i$-sphere $S^{i}$ becomes an $i$-dimensional affine planes in $E^{n}$ if $x \in S^{i}$, or a round Euclidean $i$-sphere in $E^{n}$ in $x$ is not in $S^{i}$. See Wilker [Wi], or Beardon [Be] for details about the inversive geometry. 
Let us introduce the following:

Definition 1.1. Given any set $X$ containing more than one point in $S^{n}$, the span of $X$, denoted by $\operatorname{sp}(X)$, is the unique minimal $i$-sphere in $S^{n}$ containing $X$ By minimal we mean any $j$-sphere containing $X$ contains $\operatorname{sp}(X)$.

If $X$ is a finite subset of $S^{n}$ containing more than one point, then $\operatorname{dim} \operatorname{sp}(X)$ $\leq|X|-2$. To see this, consider the Euclidean model of $S^{n}$ with infinity a point $x$ in $X$. Then $\operatorname{sp}(X)-\{x\}$ is a linear span of $X-\{x\}$ in the Euclidean space. Thus the result follows from the corresponding dimension estimate in the Euclidean space.

Inversive geometry is fundamental to the classical geometries. Indeed, the spherical geometry $\left(S^{n}, O(n+1)\right)$ is isomorphic (Klein's sense) to

$$
\left(S^{n},\left\{g \in \operatorname{Mob}\left(S^{n}\right) \mid g \theta=\theta g\right\}\right)
$$

where $\theta$ is a fixed point free involution in $\operatorname{Mob}\left(S^{n}\right) \quad(\theta$ is a conjugate of the antipodal map). An $i$-sphere $S^{i}$ in $S^{n}$ is totally geodesic if and only if $\theta\left(S^{i}\right)=S^{i}$.

Lemma 1.1. (a) Suppose $S^{i}$ is an $i$-sphere in $S^{n}$. Then $\theta\left(S^{i}\right) \cap S^{i} \neq \varnothing$ implies $\theta\left(S^{i}\right)=S^{i}$.

(b) Suppose $f \in \operatorname{Mob}\left(S^{n}\right)$ such that $\theta f(z)=f \theta(z)$ (equation in $z$ ) holds for two points distinct $x, y$ with $\theta(x) \neq y$. Then $f \in \operatorname{Centralizer}(\theta)$.

(c) Suppose $S_{1}, S_{2}, \ldots, S_{n}$ are $n(n-1)$-spheres in $S^{n}$ with $\theta\left(S_{i}\right)=S_{i}$ for all $i$. Then

$$
S_{1} \cap S_{2} \cap \cdots \cap S_{n} \neq \varnothing .
$$

Proof. Since any fixed point free involution in $\operatorname{Mob}\left(S^{n}\right)$ is conjugate (by a Möbius transformation) to the antipodal map $A: S^{n} \rightarrow S^{n}$ sending $x$ to $-x$, it suffices to show the lemma for $\theta=A$.

(a) Let $P$ be the $(i+1)$-affine subspace in $E^{n+1}$, such that $S^{i}=P \cap S^{n}$. Then $A\left(S^{i}\right) \cap S^{i} \neq \varnothing$ implies $A(P) \cap P \neq \varnothing$. Let $x \in P$ such that $A(x)=-x \in P$. Then $o=(x+A(x)) / 2 \in P$. Hence $A(P)=P$. Consequently, $\mathrm{A}\left(S^{i}\right)=S^{i}$.

(b) By composing $f$ with a spherical isometry, we may assume that $f(x)=x$ for the point $x$. Then $f(A(x))=A(f(x))=A(x)$. Consider the Euclidean model of $S^{n}$ with $x$ as the infinity, and $A(x)$ as the origin. $f$ becomes a linear similarity map, i.e.,

$$
f(z)=k g(z), \quad z \in E^{n},
$$

where $g \in O(n), k \in R^{+}$. The antipodal map $A$ is now given by

$$
A(z)=-z /\|z\|^{2}, \quad z \in E^{n} .
$$

Clearly, $A h=h A$ for all $h \in O(n)$. Thus, it suffices to show $k=1$ in order to show $f \in \operatorname{Centralizer}(A)$. Now by the assumption, $y \neq x, A(x)$, and

$$
A f(y)=f A(y) \text {. }
$$

Taking norm in (4) and using the formulas (2), (3), we conclude that $k=1$.

(c) Let $P_{i}$ be the $n$-dimensional linear subspaces of $E^{n+1}$, (in which $S^{n}$ is the unit sphere) such that $P_{i} \cap S^{n}=S_{i}$. Then the dimension formula shows

$$
\operatorname{dim}\left(P_{1} \cap \cdots \cap P_{n}\right) \geq 1 \text {. }
$$


On the other hand, $S_{1} \cap \cdots \cap S_{n}$ contains $\left(P_{1} \cap \cdots \cap P_{n}\right) \cap S^{n}$. Thus the result follows. Q.E.D.

The hyperbolic geometry $\left(H^{n}\right.$, iso $\left.\left(H^{n}\right)\right)$ can be described as

$$
\left(\operatorname{int}\left(D^{n}\right),\left\{g \in \operatorname{Mob}\left(S^{n}\right) \mid g\left(D^{n}\right)=D^{n}\right\} \text { restricted to } \operatorname{int}\left(D^{n}\right)\right)
$$

where $D^{n}$ is an $n$-ball in $S^{n}$ with $\partial D^{n}$ and $(n-1)$-sphere. The totally geodesic $i$-dimensional submanifolds $N^{i}$ of $H^{n}$ are those $i$-dimensional submanifold whose span are of $i$-dimensional and orthogonal to $\partial D^{n}$. Lastly, the similarity geometry $\left(R^{n}, \operatorname{sim}\left(R^{n}\right)\right)$ is

$$
\left(S^{n}-\{x\} \text {, isotropy }\left(\operatorname{Mob}\left(S^{n}, x\right)\right) \text { restricted to } S^{n}-\{x\}\right) \text {. }
$$

The affine spaces in $R^{n}$ are the spheres in $S^{n}$ containing $x$.

Let us now introduce the basic notion of this note.

Definition 1.2. An $n$-simplex $A^{n} \subset S^{n}$ with $(n-1)$-faces $A_{1}, \ldots, A_{n+1}$ is called a conformal simplex if the following two conditions are satisfied:

(a) $A^{n}$ is smooth, i.e., there exists a neighborhood $U$ of $A^{n}$ in $S^{n}$ and a diffeomorphism $\phi: U \rightarrow R^{n}$ such that $\phi\left(A^{n}\right)$ is a Euclidean $n$-simplex.

(b) $\operatorname{sp}\left(A_{i}\right)$ is of $(n-1)$-dimensional for all $i$, i.e., all $(n-1)$-faces lie in some $(n-1)$-spheres.

Two conformal $n$-simplices $A^{n}$ and $B^{n}$ are called equivalent if there exists $h \in \operatorname{Mob}\left(S^{n}\right)$ such that $h\left(A^{n}\right)=B^{n}$.

Note that a Möbius simplex can be subdivided (barycentric subdivision) into Möbius simplices.

A Euclidean simplex $A^{n}$ in $R^{n}$ (taken as the Euclidean model of $S^{n}$ with $x$ as infinity as in (3)) is a conformal $n$-simplex such that $x \in \bigcap_{i=1}^{n+1} \operatorname{sp}\left(A_{i}\right)$ and $x$ is not in $A^{n}$. A spherical simplex $A^{n}$ in $\left(S^{n}, O(n+1)\right)$ considered as (1) is a conformal $n$-simplex in $S^{n}$ such that $\theta\left(\operatorname{sp}\left(A_{i}\right)\right)=\operatorname{sp}\left(A_{i}\right)$ for all $i$. A hyperbolic simplex $A^{n}$ in $\left(H^{n}\right.$, Iso $\left(H^{n}\right)$ ) considered as (2) is a conformal $n$-simplex in $S^{n}$ such that $\operatorname{sp}\left(A_{i}\right) \perp \partial D^{n}$ for all $i$, and $A^{n} \cap \partial D^{n}=\varnothing$.

The following lemma establishes the basic properties of conformal simplices.

Lemma 1.2. Suppose $A^{n}$ is a conformal $n$-simplex $(n \geq 2)$ with $(n-1)$-faces $A_{1}, \ldots, A_{n+1}$, then,

(a) any $n$ members of $\left\{\mathrm{sp}\left(A_{1}\right), \ldots, \mathrm{sp}\left(A_{n+1}\right)\right\}$ form a normal intersection family, i.e., for any index set, $1 \leq i_{1}<\cdots<i_{r} \leq n+1,(r \leq n-1)$, and $j$, $1 \leq j \leq n+1, j \neq i_{k}$, for all $k, \operatorname{sp}\left(A_{j}\right)$ intersects $\bigcap_{k=1}^{r} \operatorname{sp}\left(A_{k}\right)$ transversely. In particular, $\bigcap_{k=1}^{r} \mathrm{sp}\left(A_{i_{k}}\right)$ is an $(n-r)$-sphere in $S^{n}$.

(b) if $A^{i}$ is an i-simplex in the ith skeleton of $A^{n}$, then $\operatorname{sp}\left(A^{i}\right)$ is an i-sphere in $S^{n}$, and $A^{i}$ is a conformal $i$-simplex in $\mathrm{sp}\left(A^{i}\right)$. In particular, each $A_{i}$ is an $(n-1)$-conformal simplex in $\operatorname{sp}\left(A_{i}\right)$.

(c) let $\left\{v_{1}, \ldots, v_{n+1}\right\}$ be the set of all vertices of $A^{n}$. Then

$$
\operatorname{dim} \operatorname{sp}\left\{v_{1}, \ldots, v_{n+1}\right\}=n-1 \text {. }
$$

(d) if $h \in \operatorname{Mob}\left(S^{n}\right), h\left(A^{n}\right)=A^{n}$, and $h$ fixed the vertices of $A^{n}$, then $h=$ id.

Proof. (a) By the definition of smooth $n$-simplices, the normality follows from the corresponding property of the Euclidean $n$-simplex. Since the transverse intersection of two spheres in $S^{n}$ is still a sphere, the second assertion follows. 
(b) Suppose $A^{i}=\bigcap_{k=1}^{n-i} A_{i_{k}}$. Then $\operatorname{sp}\left(A^{i}\right) \subset \cap_{k=1}^{n-i} \operatorname{sp}\left(A_{i_{k}}\right)$. The latter is an $i$-sphere by (a). On the other hand, $\operatorname{dim} \operatorname{sp}\left(A^{i}\right) \geq \operatorname{dim} A^{i}=i$. Thus $\operatorname{sp}\left(A^{i}\right)=$ $\bigcap_{k=1}^{n-i} \operatorname{sp}\left(A_{i_{k}}\right)$ is an $i$-sphere.

Any $(i-1)$-face $A^{i-1}$ of $A^{i}$ is also an $(i-1)$-simplex in the $(i-1)$ th skeleton of $A^{n}$. Thus $\operatorname{sp}\left(A^{i-1}\right)$ is an $(i-1)$-sphere in $\operatorname{sp}\left(A^{i}\right)$. Since the span of $A^{i-1}$ in $\operatorname{sp}\left(A^{i}\right)$ is the same as the span of $A^{i-1}$ in $S^{n}$, this shows that $A^{i}$ is a conformal $i$-simplex in $\mathrm{sp}\left(A^{i}\right)$ by definition. (That $A^{i}$ is a smooth $i$-simplex follows from the differential topology.)

(c) Note that $\operatorname{dim} \operatorname{sp}\left\{v_{1}, \ldots, v_{n+1}\right\} \leq n+1$ by the remark after Definition 1.1. Thus, it suffices to prove the inequality in the other direction. We prove this by induction on $n$. If $n=2$, the result is trivial, since any conformal triangle has three vertices. Assume the result holds for $n-1$. Then, in the case $n$, by induction hypothesis, we have $\operatorname{dim} \operatorname{sp}\left\{v_{1}, \ldots, v_{n+1}\right\} \geq(n-2)$. If the conclusion fails, then the above equality holds. For any $(n-1)$-face $A_{i}$ of $A^{n}$, let $X$ be the vertex set of $A_{i}$. By induction hypothesis, and (b), $\operatorname{dim} \operatorname{sp}(X)=n-2$. On the other hand, $\operatorname{sp}(X) \subset \operatorname{sp}\left\{v_{1}, \ldots, v_{n+1}\right\}$. Thus

$$
\operatorname{sp}(X)=\operatorname{sp}\left\{v_{1}, \ldots, v_{n+1}\right\} .
$$

Since $\operatorname{sp}(X) \subset \operatorname{sp}\left(A_{i}\right)$, we have then, $\operatorname{sp}\left\{v_{1}, \ldots, v_{n+1}\right\} \subset \operatorname{sp}\left(A_{i}\right)$ for all $(n-1)$-faces $A_{i}$ of $A^{n}$. This contradicts the normal intersection property of $\left\{\operatorname{sp}\left(A_{1}\right), \ldots, \operatorname{sp}\left(A_{n+1}\right)\right\}$.

(d) Since $h$ fixes the vertices of $A^{n},\left.h\right|_{\mathrm{sp}\left\{v_{1}, \ldots, v_{n+1}\right\}}=\mathrm{id}$. By (c),

$$
\operatorname{sp}\left\{v_{1}, \ldots, v_{n+1}\right\}
$$

is an $(n-1)$-sphere. Thus $h$ is the identity or the inversion on the $(n-1)$ sphere. Now $h\left(A^{n}\right)=A^{n}$ implies $h=$ id. Q.E.D.

Definition 1.3 (Vertex cone). Suppose $A^{n}$ is a conformal $n$-simplex in $S^{n}$, and suppose $A_{1}, \ldots, A_{n}$ are $n$ of the $n+1$ codimension 1 faces of $A^{n}$. Let $v$ be the vertex $\bigcap_{i=1}^{n} A_{i}$ of $A^{n}$. Then the vertex cone of $A^{n}$ at the vertex $v$, denoted by cone $\left(A^{n}, v\right)$ is the closure of the connected component of $S^{n}-\bigcup_{i=1}^{n} \operatorname{sp}\left(A_{i}\right)$ which contains int $\left(A^{n}\right)$.

Let $v^{\prime}=\bigcap_{i=1}^{n} \operatorname{sp}\left(A_{i}\right)-\{v\}$, and consider the Euclidean model of $S^{n}$ with $v^{\prime}$ as infinity. Then the interior $\operatorname{int}\left(\operatorname{cone}\left(A^{n}, v\right)\right)$ is the open Euclidean cone region bounded by the $n(n-1)$-affine spaces $\operatorname{sp}\left(A_{1}\right), \ldots, \operatorname{sp}\left(A_{n}\right)$. If $A_{n+1}$ is the remaining face, then $A^{n}$ is the closure of the complement of $\operatorname{cone}\left(A^{n}, v\right)-$ $A_{n+1}$ which contains $\operatorname{int}\left(A^{n}\right)$.

Lemma 1.3. Suppose $A^{n}$ is a conformal $n$-simplex in $S^{n}$ with $(n-1)$-faces $A_{1}, \ldots, A_{n+1}$. Then one and only one of the following assertions holds:

(a) there is a unique point $x \in S^{n}$ such that $x \in \operatorname{sp}\left(A_{i}\right)$ for all $i . A^{n}$ is called a similarity simplex. Furthermore, if $x \notin A^{n}, A^{n}$ is called a Euclidean simplex, otherwise $x \in A^{n}, A^{n}$ is called an exceptional Euclidean simplex.

(b) there is a unique $(n-1)$-sphere $S^{n-1} \subset S^{n}$ such that $\operatorname{sp}\left(A_{i}\right) \perp S^{n-1}$ for all $i . A^{n}$ is called a generalized hyperbolic simplex. If $A^{n} \cap S^{n-1}=\varnothing, A^{n}$ is called hyperbolic, otherwise, $A^{n}$ is called an exceptional hyperbolic simplex.

(c) there is a unique fixed point free involution $\theta \in \operatorname{Mob}\left(S^{n}\right)$ such that $\theta\left(\operatorname{sp}\left(A_{i}\right)\right)=\operatorname{sp}\left(A_{i}\right)$ for all $i . A^{n}$ is called a spherical simplex. (See Figure 1.1.) 

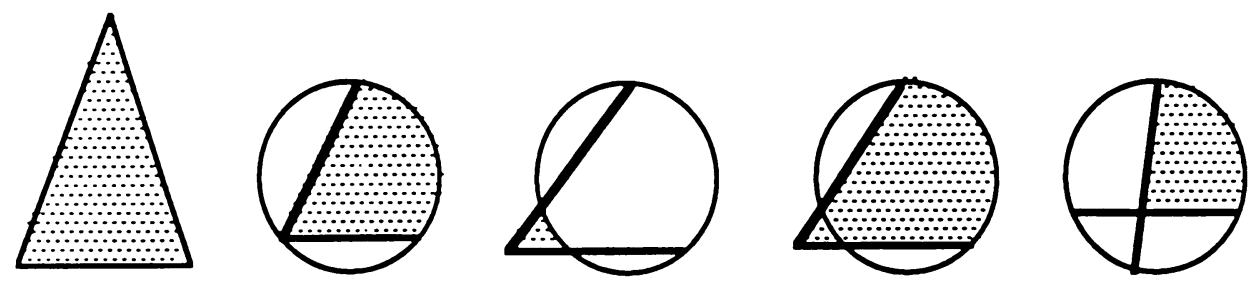

2-dimensional Möbius triangles
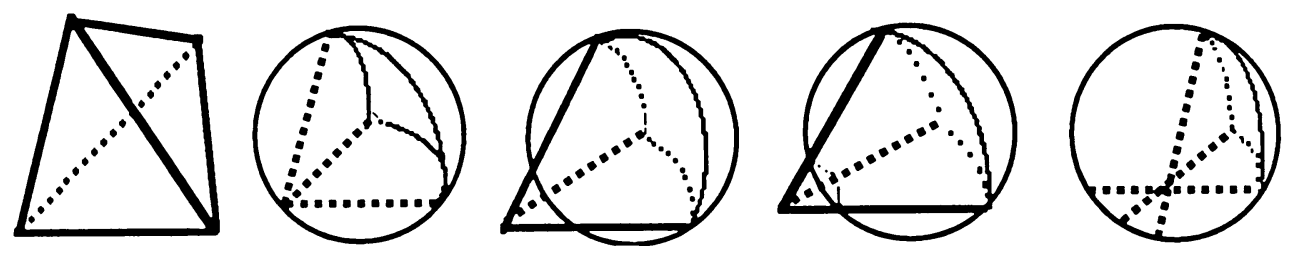

3-dimensional Möbius simplexes

FIGURE 1.1

Proof. Let $v$ be a vertex of $A^{n}$ formed by $n$ codimension one faces $A_{1}, \ldots, A_{n}$ of $A^{n}$. By Lemma 1.2(a), $\bigcap_{i=1}^{n} \operatorname{sp}\left(A_{i}\right)$ is a zero sphere $\left\{v, v^{\prime}\right\}$. Consider the Euclidean model $E^{n}$ of $S^{n}$ with $v^{\prime}$ as infinity. Thus $\operatorname{sp}\left(A_{1}\right), \ldots, \operatorname{sp}\left(A_{n}\right)$ become affine spaces intersecting at $v$. There are now two possibilities:

(I) $v^{\prime} \in \operatorname{sp}\left(A_{n+1}\right)$ or

(II) $v^{\prime} \notin \mathrm{sp}\left(A_{n+1}\right)$

where $A_{n+1}$ is the remaining $(n-1)$-face.

Case (I). Take $x=v^{\prime}$, then $x \in \operatorname{sp}\left(A_{i}\right)$ for all $i$. Now all $P_{i}=\operatorname{sp}\left(A_{i}\right)-$ $\left\{v^{\prime}\right\}$ are planes in $E^{n}$, and any $n$ members of $\left\{P_{1}, \ldots, P_{n+1}\right\}$ form a normal intersection family.

Claim. $\bigcap_{i=1}^{n+1} P_{i}=\varnothing$ in $E^{n}$.

Otherwise, any component of $E^{n}-\bigcup_{i=1}^{n+1} P_{i}$ has only two vertices due to the fact that $\bigcap_{i=1}^{n+1} P_{i}$ is one point (Lemma 1.2(a)). Therefore $P_{1}, \ldots, P_{n+1}$ cannot bound the conformal simplex.

It follows that $\bigcap_{i=1}^{n+1} \operatorname{sp}\left(A_{i}\right)=\{x\}$, i.e., $x$ is unique.

Now these planes $\left\{P_{1}, \ldots, P_{n+1}\right\}$ cut $E^{n}$ into $2^{n}$ chambers. One of them, the bounded component, say $B^{n}$, is a Euclidean simplex. $n+1$ of the rest of these chambers (the complement of $B^{n}$ in any of its vertex cones) are still conformal simplices with a vertex at the infinity. All the remaining chambers have fewer than $n+1$ vertices, see Figure 1.2. Thus in this case, $A^{n}$ is Möbius equivalent to a Euclidean simplex (in the case $x \notin A^{n}$ ), or is Möbius equivalent to the complement of a Euclidean simplex in one of its vertex cones (in the case $\left.x \in A^{n}\right)$.

Case (II). Since $v^{\prime} \notin \mathrm{sp}\left(A_{n+1}\right)$, there is an $n$-ball $D^{n}$ in $E^{n}$ such that $\partial D^{n}=$ $\operatorname{sp}\left(A_{n+1}\right)$. There are now three subcases:
(a) $v \in \partial D^{n}$,
(b) $v \notin D^{n}$
(c) $v \in \operatorname{int}\left(D^{n}\right)$. 


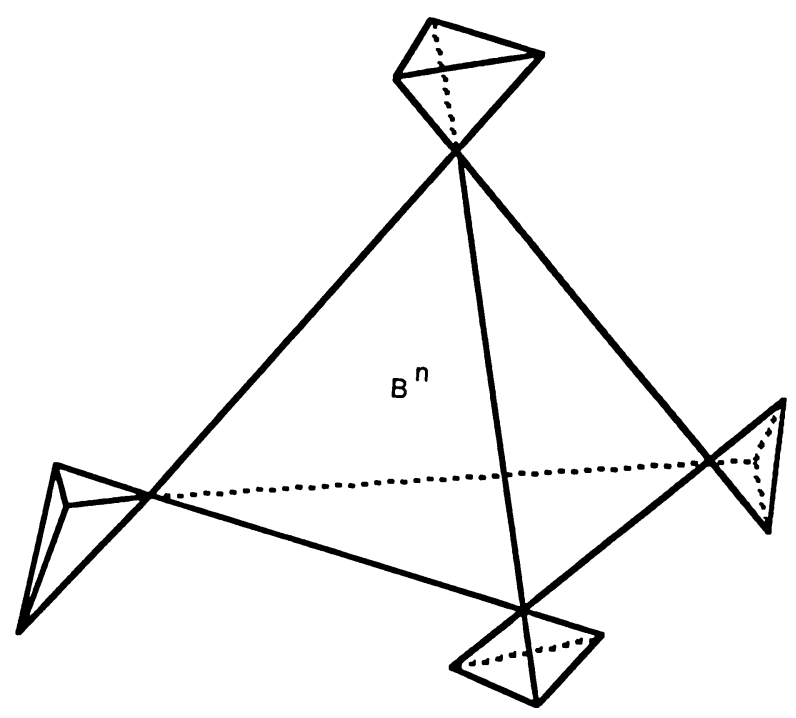

FIGURE 1.2

In the subcase (a), $v \in \bigcap_{i=1}^{n+1} \operatorname{sp}\left(A_{i}\right)$. By considering the new Euclidean model with $v$ as the infinity, we convert the case into the previous Case (I).

In the subcase (b), consider the family of all $(n-1)$-spheres centered at $v$ in the Euclidean space $E^{n}$. There is a unique member of the family, say $S^{n-1}$, such that $S^{n-1} \perp \partial D^{n}$. Clearly, by the construction, $S^{n-1} \perp \operatorname{sp}\left(A_{i}\right)$ for all $i=1,2, \ldots, n$. Therefore we arrive at the generalized hyperbolic case. One can, furthermore, classify the Möbius equivalence classes of $A^{n}$ as follows. If $S^{n-1} \cap A^{n}=\varnothing$, then $A^{n}$ lies in the interior of the ball bounded by $S^{n-1}$ in $E^{n}$. Thus by (5), $A^{n}$ is Möbius equivalent to a hyperbolic simplex. If $S^{n-1} \cap A^{n} \neq \varnothing$, then $S^{n-1} \cap \operatorname{cl}\left(\operatorname{cone}\left(A^{n}, v\right)-A^{n}\right)=\varnothing$. To see this, we need the following.

Lemma 1.4. Under the above assumption that $S^{n-1} \cap A^{n} \neq \varnothing$, we have

(a) $S^{n-1} \cap A_{n+1}=\varnothing$,

(b) $S^{n-1} \cap A_{i} \neq \varnothing, i=1,2, \ldots, n$.

Proof. cone $\left(A^{n}, v\right) \cap \operatorname{sp}\left(A_{n+1}\right)$ consists of two disjoint $(n-1)$-simplices: $A_{n+1}$ and $A_{n+1}^{\prime}$. The inversion in $S^{n-1}$ carries $A_{n+1}$ to $A_{n+1}^{\prime}$. Thus (a) follows. To be more precisely, given any point $x \in A_{n+1}$, let $x^{\prime} \in A_{n+1}^{\prime}$ be the point in $\operatorname{sp}\left(A_{n+1}\right)$ such that $v, x, x^{\prime}$ collinear in the Euclidean model $E^{n}$. Then the map sending $x$ to $x^{\prime}$ is induced by the inversion in $S^{n-1}$. In particular, the segment $x x^{\prime}$ intersects $S^{n-1}$ exactly in one point. By taking $x$ in the $(n-2)$-face $A_{i} \cap A_{n+1}$, one shows that the intersection of the segment $x x^{\prime}$ with $S^{n-1} \subset S^{n-1} \cap A_{i}$ for all $i=1,2, \ldots, n$. Hence $S^{n-1} \cap A_{i} \neq \varnothing$, for $i=1,2, \ldots, n$. Q.E.D.

Since $S^{n-1} \cap \operatorname{cl}\left(\operatorname{cone}\left(A^{n}, v\right)-A^{n}\right)=\varnothing$, we see that $\operatorname{cl}\left(\operatorname{cone}\left(A^{n}, v\right)-A^{n}\right)$ is a smooth simplex, and is a hyperbolic $n$-simplex by the case treated above. Therefore, $A^{n}$ is the complement of a hyperbolic $n$-simplex in one of its vertex cones. Furthermore, $\operatorname{cl}\left(\operatorname{cone}\left(A^{n}, v\right)-A^{n}\right)$ is a conformal $n$-simplex if $A^{n}$ is hyperbolic. Thus, exceptional hyperbolic simplices are exactly the closure of the complement of a hyperbolic $n$-simplices in one of their vertex cones. 
We now prove that $S^{n-1}$ is unique. Since there are no affine $(n-1)$-spaces in the Euclidean $n$-space orthogonal to $n$ normal intersecting affine $(n-1)$-spaces, $v^{\prime} \notin S^{n-1}$. On the other hand, an $(n-1)$-sphere orthogonal to $n$ normally intersecting affine $(n-1)$-spaces $\operatorname{sp}\left(A_{1}\right), \ldots, \operatorname{sp}\left(A_{n}\right)$ in the Euclidean space $E^{n}$ has to be centered at the $\bigcap_{i=1}^{n} \operatorname{sp}\left(A_{i}\right)-\{\infty\} \quad\left(\infty=v^{\prime}\right)$. Therefore, the result follows.

In the last subcase (c), let us consider the spherical model of the inversive geometry $S^{n}=\left\{x \in E^{n+1} \mid\|x\|=1\right\}$. Let $D^{n+1}$ be the unit ball, $\partial D^{n+1}=S^{n}$, and let $S_{i}$ be the unique $n$-sphere or $n$-linear subspace in $E^{n+1}$ orthogonal to $S^{n}$ such that $S_{i} \cap S^{n}=\operatorname{sp}\left(A_{i}\right), i=1,2, \ldots, n+1$. Then $\left\{S_{1}, \ldots, S_{n+1}\right\}$ form a normal intersection family (Lemma 1.2) in $E^{n+1} \cdot \bigcap_{i=1}^{n+1} S_{i}$ is a zero sphere disjoint from $S^{n}$ and is invariant under the inversion on $S^{n}$ in $E^{n+1}$ since each $S_{i}$ is invariant under the inversion in $S^{n}$. Thus, $D^{n+1} \cap \bigcap_{i=1}^{n+1} S_{i}$ is a point $p$ in the interior of $D^{n+1}$. Let $\bar{h}$ be the Möbius transformation of $E^{n+1}$ leaving $D^{n+1}$ invariant such that $\bar{h}(p)=O$, the origin of $E^{n+1}$. Then each of $\bar{h}\left(S_{i}\right)$ is still orthogonal to $S^{n}$ and contains the origin $O$, for $i=1,2, \ldots, n+1$. This implies $\bar{h}\left(S_{i}\right)$ is an affine hyperplane in $E^{n+1}$. Thus, $\bar{h}\left(S_{i}\right) \cap S^{n}$ is a great sphere in $S^{n}$, i.e.,

$$
A\left(\bar{h}\left(S_{i}\right) \cap S^{n}\right)=\bar{h}\left(S_{i}\right) \cap S^{n}
$$

where $A$ is the antipodal map in $E^{n+1}$. Let $h=\left.\bar{h}\right|_{s^{n}}$ be the Möbius transformation in $S^{n}$. Then $\theta=h^{-1} A h$ is the fixed point free involution of $S^{n}$ such that $\theta\left(\operatorname{sp}\left(A_{i}\right)\right)=\operatorname{sp}\left(A_{i}\right)$. Note that the closure of the complement of a spherical simplex in its vertex cone is still a spherical simplex with respect to the same fixed point free involution.

To complete the proof, we now show that $\theta$ is unique. Suppose $\theta^{\prime}$ is another fixed point free involution in $\operatorname{Mob}\left(S^{n}\right)$ such that $\theta^{\prime}\left(\operatorname{sp}\left(A_{i}\right)\right)=\operatorname{sp}\left(A_{i}\right)$ for all $i$. Write $\theta^{\prime}=g^{-1} \theta g$ for some $g \in \operatorname{Mob}\left(S^{n}\right)$. Thus,

$$
\theta g\left(\operatorname{sp}\left(A_{i}\right)\right)=g\left(\operatorname{sp}\left(A_{i}\right)\right),
$$

for all $i$. However (7) implies that $\theta g(x)=g \theta(x)$ holds for all vertices of $A^{n}$. Lemma 1.2(d) shows that $g \theta=\theta g$, i.e., $\theta^{\prime}=\theta$. Q.E.D.

For an exceptional Euclidean simplex $A^{n}$ with $(n-1)$-faces $A_{1}, \ldots, A_{n+1}$ the vertex $v=\bigcap_{i=1}^{n+1} \operatorname{sp}\left(A_{i}\right)$ is called the exceptional vertex. Note that in this case $\operatorname{cl}\left(\operatorname{cone}\left(A^{n}, v\right)-A^{n}\right)$ is a Euclidean simplex. Similarly, for an exceptional hyperbolic simplex $A^{n}$, the vertex $v$ such that $v$ and the remaining $n$ vertices of $A^{n}$ lie in the different component of $S^{n-1}$ (the sphere orthogonal to all $\operatorname{sp}\left(A_{i}\right)$ is called the exceptional vertex. Again, $\operatorname{cl}\left(\operatorname{cone}\left(A^{n}, v\right)-A^{n}\right)$ is a hyperbolic simplex if $v$ is exceptional.

Corollary 1.5. $A^{n}$ is a conformal $n$-simplex with $(n-1)$-faces $A_{1}, \ldots, A_{n+1}$ in $S^{n}$, then each $A_{i}$ is also a conformal $(n-1)$-simplices in $\operatorname{sp}\left(A_{i}\right)$ having the same type (similarity, generalized hyperbolic, and spherical) as $A^{n}$ has. Furthermore, one has:

(a) if $A^{n}$ is Euclidean, then all $A_{i}$ 's are Euclidean;

(b) if $A^{n}$ is exceptional Euclidean, then the $(n-1)$-face opposite to the exceptional vertex is Euclidean, all the remaining $A_{i}$ 's are exceptional Euclidean with the exceptional vertex $v$; 
(c) if $A^{n}$ is hyperbolic, then all $A_{i}$ 's are hyperbolic;

(d) if $A^{n}$ is exceptional hyperbolic, then the $(n-1)$-face opposite to the exceptional vertex $v$ is hyperbolic, all the remaining $A_{i}$ are exceptional hyperbolic with $v$ as the exceptional vertex;

(e) if $A^{n}$ is spherical, then all $A_{i}$ 's are spherical.

Proof. The statement follows easily from the proof of Lemma 1.3 and the observation that $x \in \operatorname{sp}\left(A_{i}\right), S^{n-1} \cap \operatorname{sp}\left(A_{i}\right)$, and $\left.\theta\right|_{\operatorname{sp}\left(A_{i}\right)}$ are the unique common point, the orthogonal $(n-2)$-sphere, and the fixed point free involution in $\operatorname{Mob}\left(\operatorname{sp}\left(A_{i}\right)\right)$ respectively, where $\operatorname{sp}\left(A_{i}\right)$ is identified with $S^{n-1}$. Q.E.D.

\section{Proof of THE THEOREM}

Recall that a Möbius manifold $M$ is an $n$-manifold modeled on the inversive geometry $\left(S^{n}, \operatorname{Mob}\left(S^{n}\right)\right)$. The Möbius triangulation of a Möbius manifold is defined to be a smooth triangulation of the manifold so that all top dimensional simplices are in some geometric coordinate charts and are Möbius simplices in the charts. Our goal is to show that a Möbius $n$-manifold $(n \geq 3)$ with a Möbius triangulation has a hyperbolic, or a spherical, or a similarity structure in the conformal class.

Let $\widetilde{M} \rightarrow M^{n}$ be the universal covering of $M^{n}, \widetilde{K}$ be the pulled back triangulation. Let $\operatorname{dev}: \widetilde{M} \rightarrow S^{n}$ be the developing map and $\rho: \pi_{1}\left(M^{n}\right) \rightarrow$ $\operatorname{Mob}\left(S^{n}\right)$ be the holonomy homomorphism. Consider an $n$-simplex $A^{n *}$ in $\widetilde{K}$. By assumption $A^{n}=\operatorname{dev}\left(A^{n *}\right)$ is a conformal $n$-simplex in $S^{n}$ with $(n-1)$ faces $A_{1}, \ldots, A_{n+1}$. Now suppose $B^{n *}$ is an $n$-simplex in $\widetilde{K}$ and $B^{n *}$ has a common $(n-1)$-face with $A^{n *}$. Let $B^{n}=\operatorname{dev}\left(B^{n *}\right)$ and $B_{1}, \ldots, B_{n+1}$ be the $(n-1)$-faces of $B^{n}$. We may assume $B_{1}=A_{1}$ and $B_{1} \cap B_{i}=A_{1} \cap A_{i}$ for $i=2,3, \ldots, n+1$. Since $n \geq 3$, and $B_{1}$ intersects $B_{i}$ transversely, we have

$$
\operatorname{sp}\left(B_{1}\right) \cap \operatorname{sp}\left(B_{i}\right)=\operatorname{sp}\left(B_{1} \cap B_{i}\right)
$$

and similarly,

Thus,

$$
\operatorname{sp}\left(A_{1}\right) \cap \operatorname{sp}\left(A_{i}\right)=\operatorname{sp}\left(A_{1} \cap A_{i}\right)
$$

for all $i=1,2, \ldots, n+1$.

By Lemma 1.3, $A^{n}$ is either one of the three types. Our goal is to show that $A^{n}$ and $B^{n}$ have the same type with respect to the same reference objects: $x$, $S^{n-1}$, or $\theta$.

Type (I). $A^{n}$ is similarity, i.e., there is a point $x \in S^{n}$ so that $x \in \operatorname{sp}\left(A_{i}\right)$ for all $i=1,2, \ldots, n+1$. By $(8), x \in \operatorname{sp}\left(B_{i}\right)$ for all $i=1,2, \ldots, n+1$. This shows that $B^{n}$ is also a similarity $n$-simplex with respect to the same point $x$. Therefore, for any $n$-simplex $C^{n *}$ in $\widetilde{K}, \operatorname{dev}\left(C^{n *}\right)$ is a similarity simplex with respect to the point $x$. This implies that $x$ is fixed by all holonomies of $M^{n}$. Indeed, let $g \in \rho\left(\pi_{1}\left(M^{n}\right)\right)$ be any holonomy element, $g\left(A^{n}\right)=\operatorname{dev}\left(C^{n *}\right)$ for some $C^{n *} \in \widetilde{K}$. Now by the uniqueness of $x$ (Lemma 1.3), we have $g(x)=$ $x$. In summary, $M^{n}$ is a conformally flat closed manifold with holonomies fixing a point $x \in S^{n}$. If $x \notin \operatorname{dev}(\widetilde{M})$, i.e., all $\operatorname{dev}\left(C^{n *}\right), C^{n *} \in \widetilde{K}$, are Euclidean, then $M^{n}$ is a similarity manifold modeled on the Euclidean model 
of the inversive geometry with $x$ as the infinity. Otherwise, $x \in \operatorname{dev}(\widetilde{M})$. By the work of D. Fried [Fr] (also see the work of Kamishima [Ka, proof of Case 2 in Theorem 3.6]), we know that $M^{n}$ is simply connected. Therefore, $M^{n}$ is conformally isomorphic to $S^{n}$. The assumption that $M^{n}$ is closed is essential to this argument.

Type (II). $\operatorname{dev}\left(A^{n *}\right)$ is spherical with respect to a fixed point free involution $\theta \in \operatorname{Mob}\left(S^{n}\right)$, i.e., $\theta\left(\operatorname{sp}\left(A_{i}\right)\right)=\operatorname{sp}\left(A_{i}\right)$ for all $i=1,2, \ldots, n+1$. By (8), we have $\theta\left(\operatorname{sp}\left(B_{1}\right) \cap \operatorname{sp}\left(B_{i}\right)\right)=\operatorname{sp}\left(B_{1}\right) \cap \operatorname{sp}\left(B_{i}\right)$ for all $i=1,2, \ldots, n+1$. Therefore, each $\operatorname{sp}\left(B_{i}\right)$ contains at least a pair of antipodal points $\{v, \theta(v)\}$. By Lemma 1.1, we have then $\theta\left(\operatorname{sp}\left(B_{i}\right)\right)=\operatorname{sp}\left(B_{i}\right)$ for all $i=1,2, \ldots, n+1$, i.e., $B^{n}$ is spherical with respect to the same fixed point free involution $\theta$. Thus for all $C^{n *} \in \widetilde{K}, \operatorname{dev}\left(C^{n *}\right)$ are spherical with respect to $\theta$.

We now show that all holonomies of $M^{n}$ commute with $\theta$. Suppose $g \in$ $\rho\left(\pi_{1}\left(M^{n}\right)\right)$ is a holonomy element, $g\left(A^{n}\right)=\operatorname{dev}\left(C^{n *}\right)$ for some $C^{n *} \in \widetilde{K}$. Thus $g\left(A^{n}\right)$ is a spherical simplex with respect to $\theta$. This implies that the equation (in $z$ ): $\theta g(z)=g \theta(z)$ is satisfied by all the vertices of $A^{n}$. By Lemma 1.1, we have $\theta g=g \theta$ i.e., all the holonomies of $M^{n}$ are isometries in the spherical geometry $\left(S^{n}, O(n+1)\right)$ considered as in (1). Hence $M^{n}$ has a spherical structure (a Riemanian metric of constant positive sectional curvature in the preferred conformally flat class). Furthermore, all simplices in $K$ are totally geodesic.

Type (III). $A^{n}$ is generalized hyperbolic with respect to an $(n-1)$-sphere $S^{n-1}$, i.e., $S^{n-1} \perp \operatorname{sp}\left(A_{i}\right)$ for all $i=1,2, \ldots, n+1$. Therefore, $S^{n-1} \perp\left(\operatorname{sp}\left(A_{1}\right) \cap\right.$ $\left.\operatorname{sp}\left(A_{i}\right)\right)$ for all $i=1,2, \ldots, n+1$. By $(8)$, we have $S^{n-1} \perp\left(\operatorname{sp}\left(B_{1}\right) \cap \operatorname{sp}\left(B_{i}\right)\right)$, for all $i=1,2, \ldots, n+1$. Thus, $S^{n-1} \perp \operatorname{sp}\left(B_{i}\right)$ for all $i=1,2, \ldots, n+1$, i.e., $B^{n}$ is also generalized hyperbolic with respect to the same $(n-1)$-sphere $S^{n-1}$. This in turn shows that for all $C^{n *} \in \widetilde{K}, \operatorname{dev}\left(C^{n *}\right)$ are generalized hyperbolic with respect to the $(n-1)$-sphere $S^{n-1}$. Again Lemma 1.3 implies that $S^{n-1}$ is fixed by all the holonomies of $M^{n}$.

We will show now that $M^{n}$ is either a hyperbolic manifold, or is $S^{n}$.

Suppose $D_{+}^{n}$ and $D_{-}^{n}$ are the two $n$-balls in $S^{n}$ bounded by $S^{n-1}$, and that $D_{+}^{n} \cap A^{n} \neq \varnothing$. There are now two subcases which may happen.

Subcase (a). For all $C^{n *} \in \widetilde{K}, \operatorname{dev}\left(C^{n *}\right)$ are hyperbolic, i.e., $\operatorname{dev}(\widetilde{M}) \cap S^{n-1}=$ $\varnothing$. Thus $\operatorname{dev}(\widetilde{M}) \subset \operatorname{int}\left(D_{+}^{n}\right)$. Therefore, $M^{n}$ admits a hyperbolic structure modeled on

$$
\left(\operatorname{int}\left(D_{+}^{n}\right),\left\{g \in \operatorname{Mob}\left(S^{n}\right) \mid g\left(D_{+}^{n}\right)=D_{+}^{n}\right\} \text { restricted to } \operatorname{int}\left(D_{+}^{n}\right)\right) .
$$

Since $M^{n}$ is assumed to be closed, the above assertion is equivalent to the existence of complete hyperbolic metric on $M^{n}$ representing the conformally flat class. Furthermore, all simplices in $K$ are totally geodesic.

Subcase (b). There is an exceptional hyperbolic $n$-simplex $\operatorname{dev}\left(A^{n *}\right), A^{n *} \in \widetilde{K}$. Put $A^{n}=\operatorname{dev}\left(A^{n *}\right)$ with the exceptional vertex $v \in \operatorname{int}\left(D_{+}^{n}\right)$. For a point $v^{*} \in \widetilde{M}$ such that $\operatorname{dev}\left(v^{*}\right)=v$, consider the open star around $v^{*}$ in $\widetilde{K}$, st $\left(v^{*}, \widetilde{K}\right)$. If an $n$-simplex $B^{n *}$ in $\operatorname{star}\left(v^{*}, \widetilde{K}\right)$ having a common $(n-1)$ face with $A^{n *}$, then $v$ is also the exceptional vertex of $B^{n}=\operatorname{dev}\left(B^{n *}\right)$ by Corollary 


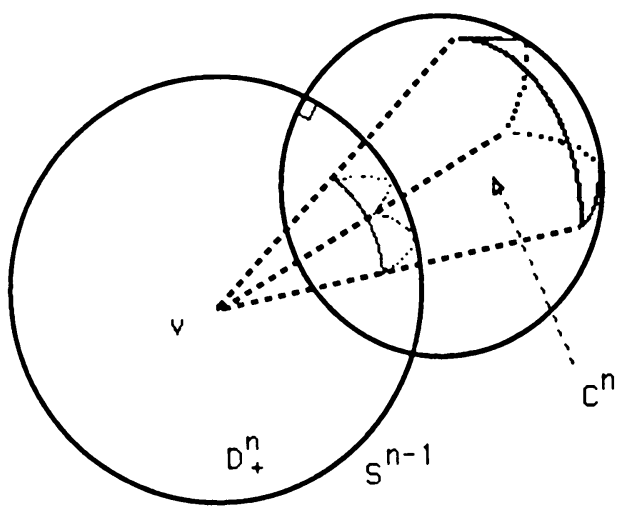

FIGURE 2.1

1.5(d). Therefore, for all $n$-simplices $C^{n *}$ in $\operatorname{st}\left(v^{*}, \widetilde{K}\right), v$ is the exceptional vertex of $C^{n}=\operatorname{dev}\left(C^{n *}\right)$. By Lemma 1.4, we have

$$
D_{+}^{n} \subset \bigcup_{C^{n *} \in \operatorname{st}\left(v^{*}, \widetilde{K}\right)} \operatorname{dev}\left(C^{n *}\right) .
$$

Hence, $\operatorname{dev}^{-1}\left(D_{+}^{n}\right) \cap \operatorname{st}\left(v^{*}, \widetilde{K}\right)$ is a geometric $n$-ball $D^{n}$ in $\widetilde{M}$. Let $p: \widetilde{M} \rightarrow$ $M^{n}$ be the covering map. The map $p$ restricted to $\left|\operatorname{st}\left(v^{*}, \widetilde{K}\right)\right|$ is an embedding onto $\left|\operatorname{st}\left(p\left(v^{*}\right), K\right)\right|$. This shows there is an $n$-ball $D_{1}^{n}$ in $M^{n}$, whose lifting to the universal covering is mapped isomorphically onto $D_{+}^{n}$ by the developing map. On the other hand, the holonomies of $M^{n}$ leave $\partial D_{+}^{n}$ invariant. Thus, by the work of W. Goldman [Go, Theorem 2.2 and Lemma 2.4], or the work of Faltings [Fa] (their result refers to two dimensional, but the proof works for an arbitrary dimension), $M^{n}$ is isomorphic to $S^{n}$. (See Figure 2.1.)

Finally, the uniqueness of the classical geometric structures in the conformally flat class is well known. Namely, for any two classical geometric structures in the same conformal class, then the two structures are either isometric (if they are hyperbolic or spherical structures), or rectilinearly equivalent (if they are the similarity structure). Q.E.D.

Remark 1. Any manifold with a classical geometric structure has a triangulation in which all simplices are totally geodesic (if the structure is hyperbolic or spherical), or rectilinear (if the structure is similarity). To see this, take a locally finite cover of the manifold by convex polytopes. The intersection of these convex polytopes are still convex polytopes and furthermore they can be triangulated into either totally geodesic simplices or rectilinear simplices according to the structure. For instance, $S^{1} \times S^{2}$ considered as $R^{3}-\{0\}$ with $x$ and $2 x$ identified has a similarity structure. Therefore, it can be triangulated into rectilinear simplices. However, these simplices may not be totally geodesic with respect to the product metric (in the conformal class).

Remark 2. If $M^{n}$ is a conformally flat manifold (not necessarily closed, or without boundary) with a Möbius triangulation, then the proof of the theorem also shows that one and only one of the following holds:

(a) there is a point in $S^{n}$ fixed by all the holonomies of $M^{n}$; 
(b) there is an $(n-1)$-sphere $S^{n}$ invariant under all the holonomies of $M^{n}$;

(c) there is a fixed point free involution $\theta \in \operatorname{Mob}\left(S^{n}\right)$ which commutes with all the holonomies of $M^{n}$.

\section{Proof of the Lemma}

We present two proofs of the configuration theorem in this section.

Lemma. Given any $n+1(n-1)$-spheres $S_{1}, \ldots, S_{n+1}$ in $S^{n}$, one of the following assertions holds:

(a) (Euclidean configuration) there is a point $x \in S^{n}$ contained in all the $S_{i}$.

(b) (Spherical configuration) there is a fixed point free involution $\theta \in \operatorname{Mob}\left(S^{n}\right)$ such that $\theta\left(S_{i}\right)=S_{i}$ for all $S_{i}$.

(c) (Hyperbolic configuration) there is an $(n-1)$-sphere $S^{n-1}$ in $S^{n}$ such that $S^{n-1} \perp S_{i}$ for all $i$.

Proof. Consider $S^{n}$ as the infinity of the hyperbolic space $H^{n+1}$. Each $S_{i}$ determines a totally geodesic hypersurface $H_{i}$ of $H^{n+1}$ which corresponds to a unit vector $V_{i}$ (up to sign) in the de Sitter space $S^{n+1,1} . V_{i}$ 's span a linear subspace $L$ of the Minkowski space $E^{n+1,1}$ (where $H^{n+1}$ and $S^{n+1,1}$ are the spheres of norm -1 and 1 respectively). The restriction of the standard bilinear form to $L$ has signature $(d-1,0)$, or $(d, 0)$, or $(d-1,1)$ where the dimension of $L$ is $d$. These correspond to the cases (a), (b), (c). To see this, the three cases imply the existence of a nonzero vector $v$ orthogonal to $L$ in the Minkowski space so that $(v, v)=0$, or $(v, v)=-1$, or $(v, v)=1$. Now these statements are the same as all $H_{i}$ 's intersect at a point at infinity, or intersect at a point in $H^{n+1}$, or orthogonal to a totally geodesic hypersurface in $H^{n+1}$.

Second proof (due to R. Edwards). Let $P_{i}$ be the affine $n$-plane in $R^{n+1}$ such that $P_{i} \cap S^{n}=S_{i}$. Let $D^{n+1}$ be the unit ball in $R^{n+1}$ bounded by $S^{n}$. There are now two cases which may occur: $\bigcap_{i=1}^{n+1} P_{i} \neq \varnothing$, or $=\varnothing$.

Case (I). $\bigcap_{i=1}^{n+1} P_{i} \neq \varnothing$. Let $x \in \bigcap_{i=1}^{n+1} P_{i}$. If $x \in S^{n}$, then (a) holds; if $x \in \operatorname{int}\left(D^{n+1}\right)$, then (b) holds. To see this, one considers the Kleinian model for the $(n+1)$-dimensional hyperbolic space int $\left(D^{n+1}\right)$. If $x \notin D^{n+1}$, then (c) holds. Indeed, let $C$ be the cone formed by $x$ and $S^{n}$. Then the boundary of $C$ intersects $S^{n}$ in an $(n-1)$-sphere $S^{n-1} . S^{n-1}$ is orthogonal to all $S_{i}$ by the construction.

Case (II). $\bigcap_{i=1}^{n+1} P_{i}=\varnothing$. Then, there is a line $L$ in $R^{n+1}$ parallel to all $P_{i}$ 's. Let $H$ be a linear $n$-space orthogonal to $L$, and let $S^{n-1}$ be the intersection $H \cap S^{n}$. Then, $S^{n-1}$ is orthogonal to all $S_{i}$ 's, i.e., (c) holds.

This ends the proof.

\section{ACKNOWLEDGMENT}

This work is essentially the author's thesis at the University of California, San Diego, The author would like to take this opportunity to thank his thesis advisor Michael Freedman for inspirations and encouragements. He also thanks B. Cox, R. Edwards, Z.-X. He, W. Thurston, and Q. Zhou for discussions and the referee for his (her) useful comments. Part of this work was carried out 
while the author was at Harvard University. He thanks the department of mathematics at Harvard University for the hospitality.

\section{REFERENCES}

[Be] A. F. Beardon, The geometry of discrete groups, Springer-Verlag, New York, 1983.

[Fa] G. Faltings, Real projective structures on Riemann surfaces, Compositio Math. 48 (1983), 223-269.

[Fr] D. Fried, Closed similarity manifolds, Comment. Math. Helv. 55 (1980), 576-582.

[Go] W. Goldman, Projective structures with Fuchsian holonomy, J. Differential Geom. 25 (1987), 297-326.

[Ka] Y. Kamishima, Conformally flat manifolds whose development maps are not surjective, Trans. Amer. MAth. Soc. 294 (1986), 607-623.

[Wi] J. B. Wilker, Inversive geometry, The Geometric Vein (C. Davis, B. Grunbaum, and F. A. Shcherk, eds.), Springer-Verlag, New York, 1981.

Department of Mathematics, University of California, Los Angeles, California 90024

Department of Mathematics, University of California, San Diego, California 92093 